\title{
Síndrome catatônica associada a transtorno bipolar misto: relato de caso e considerações terapêuticas
}

Catatonic syndrome associated with mixed bipolar disorder: a case report and therapeutic considerations

Sr. Editor,

A catatonia foi descrita pela primeira vez em 1874 por Kahlbaum, que a caracterizou como um distúrbio motor específico associado a diferentes transtornos psiquiátricos. ${ }^{1}$ Posteriormente, Kraepelin e Bleuler restringiram a catatonia a um subtipo específico da esquizofrenia. No entanto, após o questionamento de diversos autores, a associação entre catatonia e outros transtornos, notadamente os transtornos de humor, foi restabelecida. ${ }^{1}$ 
Relatamos um caso de uma mulher com episódio misto, baseado nos critérios da CID-10 (F31-6), ${ }^{2}$ e sinais compatíveis com síndrome catatônica, segundo os critérios enumerados por Fink e Taylor ( dois ou mais sintomas catatônicos, em período de uma hora, em pelo menos duas ocasiões). ${ }^{1}$ Embora dados da literatura apontem que episódios catatônicos sejam relacionados à mania mista, ${ }^{3}$ esta é a primeira descrição da associação de síndrome catatônica ao episódio misto por autores brasileiros.

Trata-se de paciente de 50 anos de idade. Foi admitida em serviço de urgência com quadro de agitação psicomotora, humor irritado, taquipsiquismo, anedonia e ideação delirante com conteúdo místico, erótico e autodepreciativo. Salientavamse estereotipias motoras, desidratação, verbigeração, palilalia e obediência automática. Não apresentava sinais parkinsonianos. Esse quadro se instalou progressivamente ao longo de 40 dias. Apresentava diagnóstico de quadro psiquiátrico há mais de 30 anos, com várias internações com sintomas de agitação e delírios, em uso crônico de antidepressivo - fluoxetina, $20 \mathrm{mg} / \mathrm{dia}$, e haloperidol, $5 \mathrm{mg} / \mathrm{dia}$.

$\mathrm{Na}$ internação, frente à agitação da paciente, prescreveramse haloperidol, $10 \mathrm{mg} / \mathrm{dia}$, biperideno, $2 \mathrm{mg} / \mathrm{dia}$, e clonazepam, $2 \mathrm{mg} /$ dia. Evoluiu ao longo da primeira semana com piora da agitação e insônia.

Diante dos sinais sugestivos de síndrome catatônica, retirouse o neuroléptico, sendo prescritos o ácido valpróico, 1.250 $\mathrm{mg} / \mathrm{dia}$, e o lorazepam, $6 \mathrm{mg} / \mathrm{dia}$. Os exames realizados foram: hemograma completo, eletrólitos, TGO, TGP, creatinina, VDRL, FTA-ABS, anti-HIV e tomografia computadorizada do encéfalo sem alterações patológicas. Após quatro dias, apresentou melhora significativa do quadro catatônico, embora tenha permanecido com humor exaltado e com ideação persecutória. Permaneceu 26 dias internada, recebendo alta hospitalar assintomática em uso de ácido valpróico, 1.250 mg/dia, e clonazepam, 4 mg/dia.
A prática clínica nos mostra que a catatonia é uma síndrome que deve ser reconhecida e tratada adequadamente para se evitar conseqüências adversas para o paciente. 0 relato ilustra bem essa condição. O uso de neuroléptico de alta potência agravou o quadro de excitação motora e sua suspensão associada à introdução de lorazepam e valproato suscitaram em melhora rápida.

A adesão ao tratamento, o diagnóstico adequado de transtorno bipolar e o uso de estabilizadores de humor são imprescindíveis na condução terapêutica de pacientes bipolares. O uso de antidepressivos deve ser evitado em episódios mistos. Embora mesmo o uso dos antipsicóticos atípicos seja controverso nos episódios de catatonia, ${ }^{1}$ há casos clínicos relatados na literatura em que o uso da olanzapina em transtorno bipolar resultou na remissão dos sintomas catatônicos associados. ${ }^{4,5}$ A literatura descreve a associação significativa entre quadro misto de humor e catatonia, ${ }^{3}$ bem como a resposta de quadros catatônicos a lorazepam e eletroconvulsoterapia. ${ }^{6} 0$ uso de antipsicóticos de alta potência deve ser evitado devido ao risco de piora de catatonia maligna e possível progressão para síndrome neuroléptica maligna.

Alexandre da Costa Val, Anderson Souza, Rodrigo Nicolato Instituto Raul Soares, Fundação Hospitalar do Estado de Minas Gerais (FHEMIG), Belo Horizonte (MG), Brasil

Antônio Lúcio Teixeira

Departamento de Clínica Médica, Faculdade de Medicina, Universidade Federal de Minas Gerais (UFMG),

Belo Horizonte (MG), Brasil

João Vinícius Salgado

Instituto Raul Soares, Fundação Hospitalar do Estado de Minas Gerais (FHEMIG), Belo Horizonte (MG), Brasil Universidade FUMEC, Belo Horizonte (MG), Brasil

Financiamento e conflito de interesses

\begin{tabular}{|c|c|c|c|c|c|c|c|}
\hline $\begin{array}{l}\text { Membro do grupo de } \\
\text { autores }\end{array}$ & $\begin{array}{l}\text { Local de } \\
\text { trabalho }\end{array}$ & $\begin{array}{l}\text { Verba de } \\
\text { pesquisa }^{1}\end{array}$ & $\begin{array}{l}\text { Outro apoio à pesquisa } \\
\text { ou educação médica } \\
\text { continuada }\end{array}$ & $\begin{array}{c}\text { Honorários de } \\
\text { palestrante }\end{array}$ & $\begin{array}{l}\text { Participação } \\
\text { acionária }\end{array}$ & $\begin{array}{l}\text { Consultor/ Conselho } \\
\text { consultivo }\end{array}$ & Outro $^{3}$ \\
\hline Alexandre Costa Val & FHEMIG & -- & -- & -- & -- & --- & --- \\
\hline Anderson Souza & FHEMIG & -- & --- & --- & -- & --- & -- \\
\hline Rodrigo Nicolato & FHEMIG & -- & -- & -- & -- & -- & --- \\
\hline Antônio Lúcio Teixeira & UFMG & --- & --- & -- & --- & --- & -- \\
\hline João Vinícius Salgado & FHEMIG & --- & --- & --- & -- & --- & -- \\
\hline
\end{tabular}

Referências

1. Fink M, Taylor MA. Catatonia: a Clinician's Guide to Diagnosis and Treatment. Cambridge, UK: Cambridge University Press; 2003.

2. Classificação de Transtornos Mentais e de Comportamento da CID-10 - Diretrizes Diagnósticas e de Tratamento para Transtornos Mentais em Cuidados Primários. Organização Mundial da Saúde. Porto Alegre: Artes Médicas; 1998.

3. Krüger S, Cooke RG, Spegg CC, Bräunig P. Relevance of the catatonic syndrome to the mixed manic episode. J Affect Disord. 2003;74(3):279-85.

4. Nicolato R, Romano-Silva MA, Correa H, dos Santos RR, Teixeira AL. Stuporous catatonia in an elderly bipolar patient: response to olanzapine. Aust N Z J Psychiatry. 2006;40(5):498.

5. Guzman CS, Myung VHMi, Wan YP. Treatment of periodic catatonia with olanzapine: a case report. Rev Bras Psiquiatr. 2007;29(4):380.

6. Bush G, Fink M, Petrides G, Dowling F, Francis A. Catatonia, II: treatment with lorazepam and eletroconvulsive therapy. Acta Psychatr Scand. 1996;93(2):137-43. 